\title{
Nanoscale functional chemistry and opto-electronic response of organic materials
}

\author{
Sean Collins ${ }^{1}$, Christial Vollmer ${ }^{2}$, Quentin Ramasse ${ }^{3}$ and Demie Kepaptsoglou ${ }^{4}$
}

${ }^{1}$ University of Leeds, United States, ${ }^{2}$ University of Munster, Germany, ${ }^{3}$ SuperSTEM Laboratory, SciTech Daresbury Campus, Daresbury WA4 4AD, United Kingdom, Darebury, United Kingdom, ${ }^{4}$ SuperSTEM Laboratory, SciTech Daresbury Campus, Daresbury WA4 4AD, United Kingdom, United States

Vibrational spectroscopies offer of the ability to interrogate chemical bonds directly. Conventionally used IR and Raman spectroscopies probe molecular vibrations and phonons in large materials volumes, capturing details of functional groups and lattice dynamics, while tip-enhanced scanning near-field implementations have enabled vibrational spectroscopy approaching $10 \mathrm{~nm}$ spatial resolution but limited to surface studies. Recent advances in electron energy loss spectroscopy in the scanning transmission electron microscope (STEMEELS) have enabled the observation of the spectral signatures of phonons and molecular vibrations at much shorter length scales. In molecular systems, where long range interactions have enabled 'aloof' spectroscopy with reduced electron beam induced damage, the broadband energy window accessible in high resolution EELS also makes it possible to detect the optical signatures from the mid-infrared to the UV energy range. However, understanding variations in bonding or optical response at the nanoscale in molecular and metalorganic solids requires measurements with higher spatial sensitivity than afforded by the simple aloof beam approach. Here, we develop strategies for high spatial and energy resolution STEM-EELS to fingerprint the optical properties and molecular vibrations of beam-sensitive organic materials at the nanoscale.

Organic and metal-organic framework (MOF) glasses show significant promise in applications from LEDs and photovoltaics to photocatalysis but their macroscopic optoelectronic properties are often only linked to microscopic optical properties and atomic structure in statistical descriptions of volumes much larger than the molecule or unit cell. In contrast, high-energy-resolution STEM-EELS provides a nanoscale fingerprint of these optical properties and of characteristic molecular vibrations in MOFs. In particular, as a result of tetrahedral ligand-field splitting at Co metal centres, $d-d$ transitions directly reflect the local coordination [1] , while intra-ligand and metal-to-ligand transitions in the vibrational signal can be mapped with nm precision to distinguish nanoscale regions of distinct coordination chemistry. [2]

Perylene diamide (PDI) represents a major class of n-type organic semiconductors exhibiting particularly long exciton lifetimes that are extremely attractive for optoelectronic applications. Their observation, however, is a great challenge due to severe damage under electron beam irradiation even at moderate rates. Here, we use STEM EELS measurements in a spatially correlated near-aloof geometry, to record spatially resolve spectra in the vicinity of structural features such as point or line defects in PDI nanobelts. The beam-positioning strategy mitigates beam damage and enables the observation of the vibrational and optical characteristics of the nanobelts and changes therein at extended defects.

Meteoritic samples with organic inclusions, such as carbonaceous chondrites, are of particular importance in the study of ancient organic compounds that may have served as precursors for the prebiotic history of the early Earth. In order to elucidate the functional chemistry of these materials and understand the complex processes of formation and consecutive alterations they might have undergone in the early solar nebula, we use monochromated STEM EELS measurements at low acceleration voltage and controlled beam current. These conditions below the $\mathrm{C}$ knock-on damage threshold and with reduced electron doses, allow for the detailed observation of the pristine chemistry of the organics; vibrational EELS measurements alongside the carbon and nitrogen near edge fine structures (ELNES) reveal the presence of different functional groups, namely aliphatic and carboxyl bonds, on a submicron scale which can be attributed to early cometary and meteoritic organic reservoirs. [3,4] 
(a)

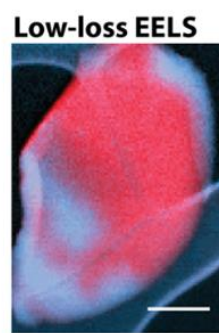

(c) Core-loss EELS

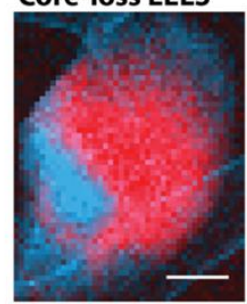

(b)
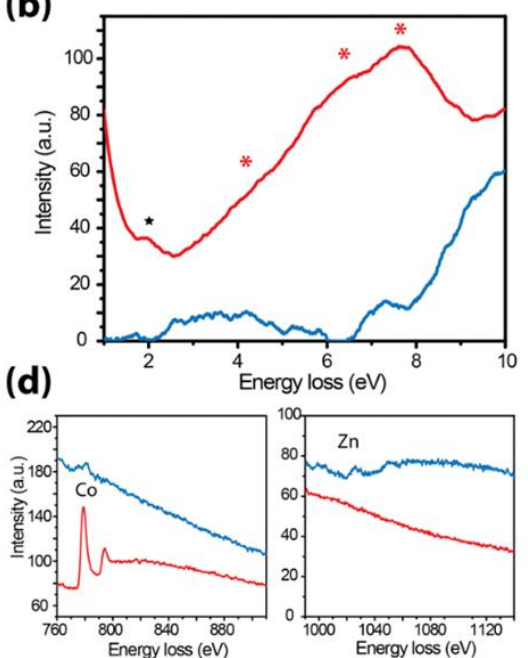

Figure 1. Figure1. Low- (a-b) and core- loss (c-d) STEM measurements of zeolitic imidazolate (ZIF) metalorganic framework glass blend. Panel (a) shows superimposed coloured maps generated by non-negative matrix factorization (NMF) applied on low loss EELS data, while the corresponding spectral factors are illustrated in (b). The method is used to directly visualise the $\mathrm{d}$-d transitions at Co metal centres (marked by * in (b)), reflecting the local tetrahedral ligand-field splitting. Panel (c) shows superimposed maps of Co and Zn of the same particle, generated by independent component analysis (ICA) from coreloss EELS data (d). The scale bars are $300 \mathrm{~nm}$. Adapted from [1].
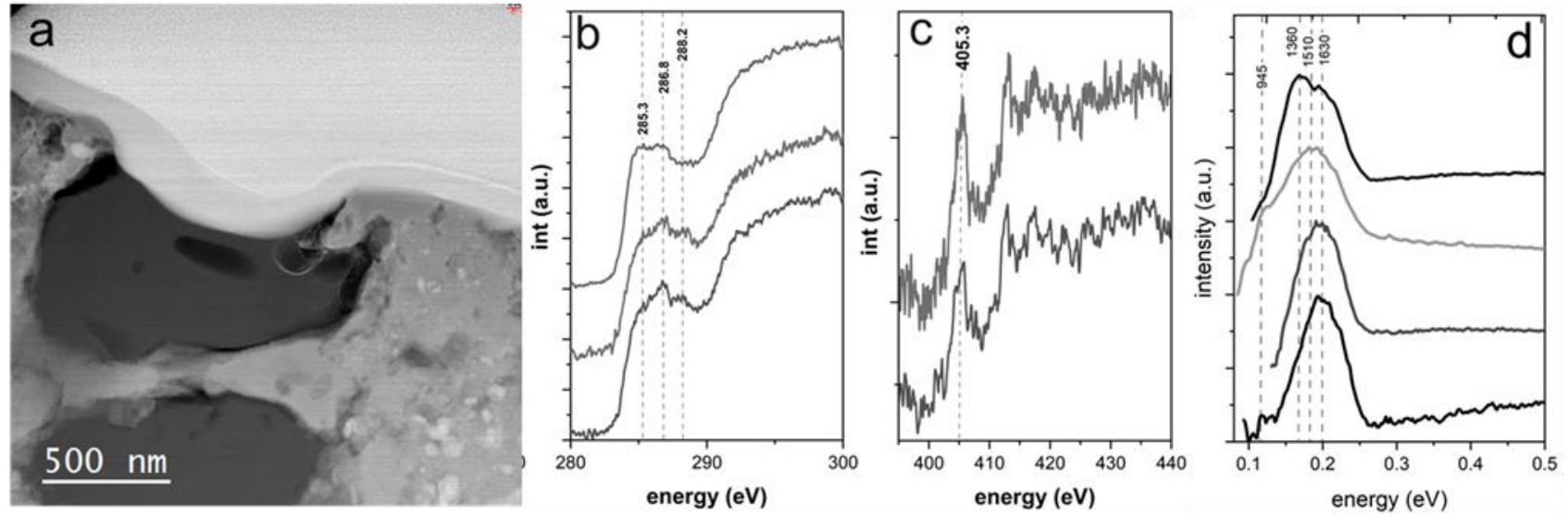

Figure 2. Figure 2. a) High Angle Annular Dark Field (HAADF) STEM image of an organic inclusion in a Renazzo type carbonaceous chondrite. (b,c) monochromated core loss measurements of the $\mathrm{C} \mathrm{K}$ and $\mathrm{N} \mathrm{K}$ ionization edges showing that the organic functional chemistry of the inclusion including ketone/aldehyde/nitrile and carboxylic bonding and possible nitrate groups and d) vibrational EELS measurements; the two major bands at around $1360 \mathrm{~cm}-1$ and $1630 \mathrm{~cm}-1$ can be identified, which relate to the D- and G-bands of amorphous carbon. The peak positions on different regions within the grain vary on a nanometer scale, most likely due to the heterogeneous nature of the organic matter. Adapted from [3].

\section{References}

[1] S. M. Collins, D. M. Kepaptsoglou et al., J. Am. Chem. Soc. 140, 17862 (2018).

[2] S. M. Collins, D. M. Kepaptsoglou et al., Nano Lett. 20, 1272 (2020).

[3] C. Vollmer, J. Leitner et-al.,Meteorit. Planet. Sci. 55, 1293 (2019). 
[4] C. Vollmer, J. Leitner et-al., Sci. Rep. 10, 20251 (2020). 